\title{
Two-body light front wave functions from general AdS/QCD models
}

\author{
Alfredo Vega ${ }^{*}$ and Miguel Angel Martin Contreras ${ }^{\dagger}$ \\ Instituto de Física y Astronomía, Universidad de Valparaíso, A. Gran Bretaña 1111, Valparaíso, Chile
}

(Received 14 May 2020; accepted 4 August 2020; published 20 August 2020)

\begin{abstract}
In this work, we consider an extension to the matching procedure proposed by Brodsky and de Teramond to obtain the two-body wave functions in the light-front formalism for holographic models. We compute the light-front wave function by considering different static dilaton fields and anti-de sitter (AdS)like geometric deformations. We also prove that this procedure holds for general AdS/QCD models in asymptotically AdS geometries.
\end{abstract}

DOI: 10.1103/PhysRevD.102.036017

\section{INTRODUCTION}

The hadronic wave function in terms of the quark and gluon degrees of freedom plays an important role in predictions for several QCD phenomena. But in the process of directly extracting this quantity some problems can arise. There are many nonperturbative approaches to obtain properties of the distribution amplitudes and hadronic wave functions from QCD. Some time ago, based on the AdS/CFT correspondence, Brodsky and de Teramond suggested a matching procedure to obtain mesonic lightfront wave functions (LFWFs) in terms of fields dual to hadrons and electromagnetic currents on the anti-de Sitter (AdS) side [1,2].

The LFWF obtained from the matching procedure was later improved by adding different ingredients to the QCD side, but its direct effects on the AdS side were not considered. For example, in Ref. [3] the authors developed a procedure to modify the LFWF by including massive quarks, which has been used widely in the literature to calculate hadron properties (see, e.g., Refs. [4-14]). Other authors introduced changes by considering the 't Hooft model [15] or looking for consistency with the Drell-YanWest relation between the large-momentum-transfer behavior observed in the nucleon electromagnetic form factors and the large- $x$ behavior of the structure functions and with the quark counting rules, producing a LFWF with an arbitrary twist [16].

Although it is well known that matrix elements of currents in AdS are related to interaction terms and are

\footnotetext{
alfredo.vega@uv.cl

†miguelangel.martin@uv.cl
}

Published by the American Physical Society under the terms of the Creative Commons Attribution 4.0 International license. Further distribution of this work must maintain attribution to the author(s) and the published article's title, journal citation, and DOI. Funded by SCOAP ${ }^{3}$. also connected to form factors (see, e.g., Refs. [1,2,17-21]), up to now it has not been studied how to establish, in general, the matching procedure that allows one to relate AdS modes with the LFWF; as we mentioned above, in all of the extensions done for the holographic LFWF the authors considered the holographic proposal with the AdS metric and quadratic dilaton as an initial ansatz and introduced changes on the QCD side without paying attention to what happens with the holographic model.

Even though the soft wall model with a quadratic dilaton and the AdS metric was a good starting point for building hadron models in a gravity frame, this model has been improved to capture more phenomenology. So far several dilatons and asymptotically AdS metrics have been considered, and we do not know how to build the LFWF associated with these models. This fact motivated us to extend the light-front holography ideas to other models.

This paper is organized as follows. In Sec. II we summarize the matching procedure which allows us to relate the AdS modes with a two-body bound-state LFWF. In Sec. III we study the large- $Q^{2}$ limit in the equation of motion (EOM) for the modes dual to photons. We further notice that in this limit we get the same equation as in the traditional quadratic dilaton, which is used for a wide variety of AdS/QCD models. This is the key ingredient that opens the door to relate the AdS modes with the two-body LFWF for several holographic models. In Sec. IV we consider four AdS/QCD models and develop their associated LFWFs. In the Appendix we discuss a relation between $g\left(x, Q^{2}\right)$, written in Eq. (10), with the Hankel transform of $J\left(z, Q^{2}\right)$. Finally, in Sec. V we present our conclusions and summarize our work.

\section{TWO-BODY WAVE FUNCTION IN HOLOGRAPHIC MODELS}

In Refs. [1,2] the authors showed that, based on the comparison of form factors calculated in the light-front 
formalism and in the AdS/QCD models, it is possible to relate bulk modes to light-front wave functions. Below we briefly discuss this matching procedure, including a generalization that will allow us to use this formalism with general AdS/QCD models.

In the light-front formalism, the electromagnetic form factor of the pion can be written as

$F\left(Q^{2}\right)=2 \pi \int_{0}^{1} d x \frac{1-x}{x} \int_{0}^{\infty} d \zeta \zeta J_{0}\left(\zeta Q \sqrt{\frac{1-x}{x}}\right) \tilde{\rho}(x, \zeta)$,

where $Q^{2}$ is the spacelike transferred momentum squared, $J_{0}$ is the Bessel function of zeroth order, and $\zeta$ is a variable defined as

$$
\zeta=\sqrt{\frac{x}{1-x}}\left|\sum_{j=1}^{n-1} x_{j} b_{j}\right|,
$$

which represents the $x$-weighted transverse impact coordinate associated with the spectator system, where $b_{j}$ is the internal distance between constituents and the sum is over the number of spectators. In the two-body case, $\zeta^{2}=x(1-x) b^{2}$.

In Eq. (1), $\tilde{\rho}(x, \zeta)$ is the effective transverse density of partons, which in the two-body case is given by

$$
\tilde{\rho}_{n=2}(x, \zeta)=\frac{\left|\tilde{\psi}_{q_{1} \bar{q}_{2}}(x, \zeta)\right|^{2}}{A^{2}(1-x)^{2}},
$$

where $A$ is a normalization constant.

Now we pay attention to form factor calculations on the gravity side in AdS/QCD models. The matrix element for the spin- $s$ current and the spin- $J$ hadrons is given by [17]

$\left\langle b\left|J^{\mu_{1} \mu_{2} \ldots \mu_{s}}\right| a\right\rangle=($ charge $)($ kinematic factor $) F_{a b}\left(Q^{2}\right)$,

where $F_{a b}\left(Q^{2}\right)$ is the form factor.

Assuming a minimal coupling, the hadronic matrix element for the electromagnetic current in asymptotically AdS spaces used in AdS/QCD models has the form [1,2]

$i g_{5} \int d^{4} x d z \sqrt{g} e^{-\phi(z)} A^{l}(x, z) \Psi *_{p^{\prime}}(x, z) \stackrel{\leftrightarrow}{\partial}_{l} \Psi_{p}(x, z)$,

where $g_{5}$ is a five-dimensional effective coupling constant and $\Psi_{p}(x, z)$ is a normalizable mode representing a hadronic state, $\Psi_{p}(x, z) \sim e^{-i p \cdot x} \Psi(z)$, with hadronic invariant mass given by $p_{\mu} p^{\mu}=M^{2}$. Additionally, it includes an electromagnetic probe polarized along Minkowski coordinates $A_{\mu}=\epsilon_{\mu} e^{-i Q \cdot x} J(Q, z), A_{z}=0$, where $J(Q, z)$ has the value 1 at zero momentum transfer. Since we are normalizing the bulk solutions to the total charge operator, and as a boundary limit, the external current is $A_{\mu}(x, z \rightarrow 0)=\epsilon_{\mu} e^{-i Q \cdot x}$. Thus, $J\left(Q^{2}=0, z\right)=J\left(Q^{2}, z=0\right)=1$.
We consider an asymptotically AdS space with a metric defined as

$$
d s^{2}=e^{2 A(z)}\left(\eta_{\mu \nu} d x^{\mu} d x^{\nu}-d z^{2}\right)
$$

where $\eta_{\mu \nu}$ is the Minkowski four-dimensional spacetime metric, $z$ is the holographic coordinate, and $A(z)$ defines the warp factor for an asymptotically AdS space, i.e., $e^{2 A(z \rightarrow 0))}=\frac{R^{2}}{z^{2}}$. Additionally, the model includes a dilaton field $\phi(z)$ that breaks the conformal invariance. With these ingredients, the corresponding expression for the form factors related to scalar hadrons in $\mathrm{AdS}$ is

$$
F\left(Q^{2}\right)=\int_{0}^{\infty} d z e^{3 A(z)-\phi(z)} \Psi(z) J\left(Q^{2}, z\right) \Psi(z),
$$

where $\Psi(z)$ and $J\left(Q^{2}, z\right)$ are the AdS modes dual to scalar hadrons and photons. These modes are the solutions of the bulk EOM in the Strum-Liouville form associated with each bulk field. The latter form factor can be transformed into the following expression:

$$
F\left(Q^{2}\right)=\int_{0}^{\infty} d z \Phi(z) J\left(Q^{2}, z\right) \Phi(z),
$$

where $\Phi(z)$ corresponds to the solutions of the EOM transformed into a Schrödinger-like form, while $J\left(Q^{2}, z\right)$ is the same solution as that used before.

In order to generalize the ideas exposed in Refs. [1,2], i.e., to put Eq. (8) in the same mathematical form as Eq. (1) -which allows us to perform a matching between AdS modes dual to hadrons and the LFWF- the key step (not considered up to now in the general AdS/QCD case) is to write the electromagnetic current as [4]

$$
J\left(Q^{2}, z\right)=\int_{0}^{1} d x g\left(Q^{2}, x\right) J_{0}\left(\zeta Q \sqrt{\frac{1-x}{x}}\right) .
$$

After putting $z=\zeta$ and considering the $x$ variable with the same physical interpretation as in Eq. (1), this allows us to compare both form factors, allowing us to establish a matching that gives a relationship between the AdS modes and the LFWF:

$$
\left|\tilde{\psi}_{q_{1} \bar{q}_{2}}(x, \zeta)\right|^{2}=A^{2} x(1-x) g\left(Q^{2}, x\right) \frac{|\Phi(\zeta)|^{2}}{2 \pi \zeta} .
$$

Here the factor $A$ is constrained by the probability condition $P_{q_{1} \bar{q}_{2}}=\int_{0}^{1} d x \int d^{2} b\left|\tilde{\psi}_{q_{1} \bar{q}_{2}}(x, b)\right|^{2} \leq 1$, with $P_{q_{1} \bar{q}_{2}}$ being the probability of finding the Fock valence state $\left|q_{1} \bar{q}_{2}\right\rangle$ in the meson $M$.

Recalling that in the two-body case we have $\zeta^{2}=x(1-x) b^{2}$, the relationship between AdS modes and the LFWF is written as follows: 


$$
\left|\tilde{\psi}_{q_{1} \bar{q}_{2}}(x, b)\right|^{2}=A^{2} \frac{\sqrt{x(1-x)}}{2 \pi b} g\left(Q^{2}, x\right)|\Phi(\sqrt{x(1-x)} b)|^{2} .
$$

In general, is not so difficult to obtain $\Phi(\zeta)$ numerically in most of the known AdS/QCD models, but obtaining $g\left(Q^{2}, x\right)$ is a problem that has not been addressed. The expression (10) has been used in AdS/QCD models, both in the hard-wall model where $g\left(Q^{2}, x\right)=1$ and in the softwall model with a quadratic dilaton. In the latter there is an extra condition, $g\left(Q^{2}, x\right)=1$, which is only achieved in the large- $Q^{2}$ case.

\section{A. Example 1: Hard-wall model}

The hard-wall model proposal considers an AdS spacetime cut by an energy scale located at $z=z_{0}$ that acts as a $D$-brane for the bulk fields. As in the original AdS/CFT correspondence, the normalizable part of the bulk field defines the hadronic modes and the non-normalizable part is connected to the Schwinger source of the hadronic operators at the conformal boundary.

In this case, the modes dual to hadrons are written as

$$
\psi(z)=C \sqrt{z} J_{L}(z \mathcal{M})
$$

where $C$ is a normalization constant, $J_{L}(z)$ is a Bessel function, and $L$ is the orbital angular momentum introduced through the relationship between the conformal dimension of modes and the scaling dimension of operators. Its eigenvalues are determined by the Dirichlet boundary conditions at $z=z_{0}$.

The EM current in this formalism is written as

$$
J(Q, z)=z Q K_{1}(z Q)
$$

where $K_{1}(z)$ is the Bessel function of the second kind.

By using these expressions in the integral form shown in Eq. (A4), we can see that $g\left(Q^{2}, \alpha\right)=g\left(Q^{2}, x\right)=1$. Therefore, we obtain the well-known integral representation

$$
J(Q, z)=z Q K_{1}(z Q)=\int_{0}^{1} d x J_{0}\left(\zeta Q \sqrt{\frac{1-x}{x}}\right) .
$$

Using this equation in Eq. (10), we obtain

$$
\tilde{\psi}_{q_{1} \bar{q}_{2}}(x, \zeta)=B \sqrt{x(1-x)} J_{L}(\zeta \mathcal{M})
$$

which is the same LFWF obtained in Ref. [1] for the hardwall model. In this case, $\zeta$ goes from zero to a maximum value $\zeta_{0}$ and $B$ is a new constant collecting the old one and some factors that appear during the calculation.

\section{B. Example 2: Traditional soft-wall model}

Here we discuss a model that considers an AdS spacetime together with a quadratic dilaton $\phi(z)=\kappa^{2} z^{2}$ that breaks the conformal invariance smoothly by introducing a scale $\kappa$.

In this case, the normalizable modes are written in terms of the associated Laguerre polynomials $L_{n}^{m}(z)$ as

$$
\psi(z)=A z^{1 / 2+L} e^{-\kappa^{2} z^{2} / 2} L_{n}^{L}\left(\kappa^{2} z^{2}\right),
$$

and the current is written in terms of the Tricomi function $U(a, b, z)$,

$$
J\left(Q^{2}, z\right)=\Gamma\left(1+\frac{Q^{2}}{4 \kappa^{2}}\right) U\left(\frac{Q^{2}}{4 \kappa^{2}}, 0, \kappa^{2} z^{2}\right) .
$$

In Appendix A 2, we consider the exact form of $g\left(Q^{2}, x\right)$ for this dilaton field. Here we will restrict ourselves to the large- $Q^{2}$ limit discussed in Refs. [2,4], with the condition $Q^{2} \gg 4 \kappa^{2}$. At this limit it is possible to observe that

$$
J\left(Q^{2}, z\right) \rightarrow z Q K_{1}(z Q),
$$

where the integral representation for $J\left(Q^{2}, z\right)$ is the same as the one used in the hard-wall case. Therefore, if we apply Eq. (10) we can obtain the an expression for the two-body wave function in the soft-wall model,

$$
\tilde{\psi}_{q_{1} \bar{q}_{2}}(x, \zeta)=B \sqrt{x(1-x)} \zeta^{L} e^{-\kappa^{2} \zeta^{2}} L_{n}^{L}\left(\kappa^{2} \zeta^{2}\right),
$$

which is the expression derived in Ref. [2] and widely used in the literature in this version, which includes massive quarks according to the prescription discussed in Ref. [3].

The fact that we consider the large- $Q^{2}$ limit to do the matching is not problematic since in the Fock expansion for the meson wave function the valence contribution is dominant at large $Q^{2}[2,4]$. Therefore, we can conclude that the expression (19) is a reasonable approach in the softwall model.

\section{GENERAL SOFT-WALL MODEL AT HIGH $Q^{2}$}

As we mentioned in the previous section, the key ingredient is to put the current in the form given by the expression (9) and then identify the $g\left(Q^{2}, x\right)$ function. As we have seen in one of the examples developed in the last section, this function is equal to one in the large- $Q^{2}$ limit for the traditional soft-wall model with a quadratic dilaton in the AdS geometry. In this section we will analyze what happens if we consider an arbitrary dilaton with a generic asymptotically AdS geometry.

To do this extension, we consider a general AdS-like warp factor given by 
TABLE I. Summary of the AdS/QCD models used to construct the LF wave function with their corresponding parameters and references.

\begin{tabular}{lcccr}
\hline \hline Model & Dilaton & Deformation & Parameters & Ref. \\
\hline 1 & $\phi_{1}(z)=\kappa^{2} z^{2}$ & $h_{1}(z)=0$ & $\kappa=0.375 \mathrm{GeV}$ & {$[24]$} \\
2 & $h_{2}(z)=\mu_{G}^{2} z^{2} \tanh \left(\frac{\mu_{G^{2}}^{2}}{\mu_{G}^{2}} z^{2}\right.$ & $h_{2}(z)=0$ & $\mu_{G}=0.370 \mathrm{GeV}$ and $\mu_{G^{2}}=0.368$ & {$[25]$} \\
3 & $\phi_{3}(z)=\kappa^{2} z^{2}+M z+\tanh \left(\frac{1}{M z}-G\right)$ & $h_{3}(z)=0$ & $\kappa=0.358 \mathrm{GeV}, M=0.083 \mathrm{GeV}$ and $G=0.082 \mathrm{GeV}$ & {$[26]$} \\
4 & $\phi_{4}(z)=0$ & $h_{4}(z)=\frac{1}{2} k z^{2}$ & $k=-0.280^{2} \mathrm{GeV}^{2}$ & {$[27]$} \\
\hline \hline
\end{tabular}

$$
A(z)=\ln \left(\frac{R}{z}\right)+h(z)
$$

where $R$ is the AdS radius and $h(z \rightarrow 0)$ is a deformation function that vanishes in the limit $z \rightarrow 0$. The EOM for the current $J\left(Q^{2}, z\right)$ reads as [22]

$$
\begin{aligned}
& \partial_{z}^{2} J\left(Q^{2}, z\right)-\left[\frac{1}{z}-\partial_{z}(h(z)-\phi(z))\right] \partial_{z} J\left(Q^{2}, z\right) \\
& \quad+Q^{2} J\left(Q^{2}, z\right)=0 .
\end{aligned}
$$

Notice that to get the LFWF we consider a matching involving the expression (8), where the modes dual to
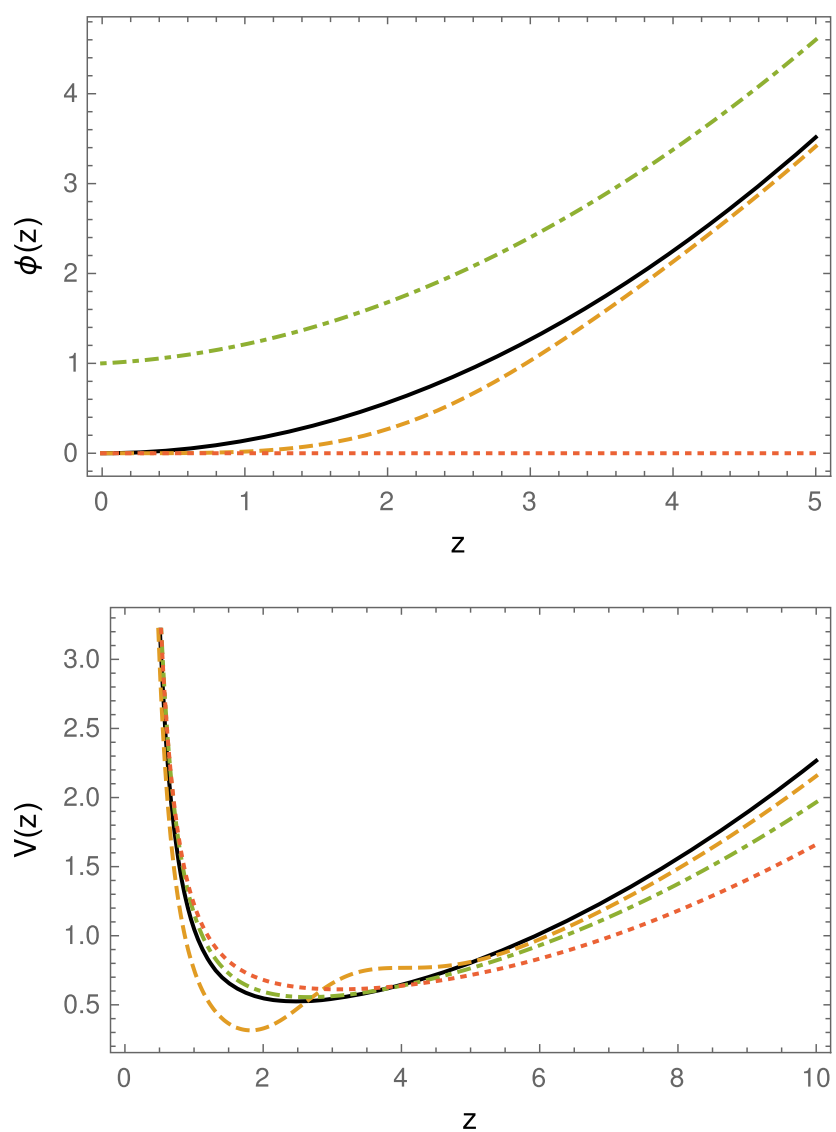

hadrons must be normalizable. Since we are interested in the current $J\left(Q^{2}, z\right)$ written in the large- $Q^{2}$ limit, holographically we can fulfill this condition with the low- $z$ limit. In other words, large $Q^{2}$ is equivalent to the limit $z \rightarrow 0$ in this context. In this limit, Eq. (21) reduces to

$$
\partial_{z}^{2} J\left(Q^{2}, z\right)-\left(\frac{1}{z}\right) \partial_{z} J\left(Q^{2}, z\right)+Q^{2} J\left(Q^{2}, z\right)=0,
$$

which is the same equation as in the hard-wall case, i.e., $g\left(Q^{2}, x\right)=1$. Recall that the asymptotically AdS condition is translated into the vanishing of the deformation function at the conformal boundary. This condition ensures the
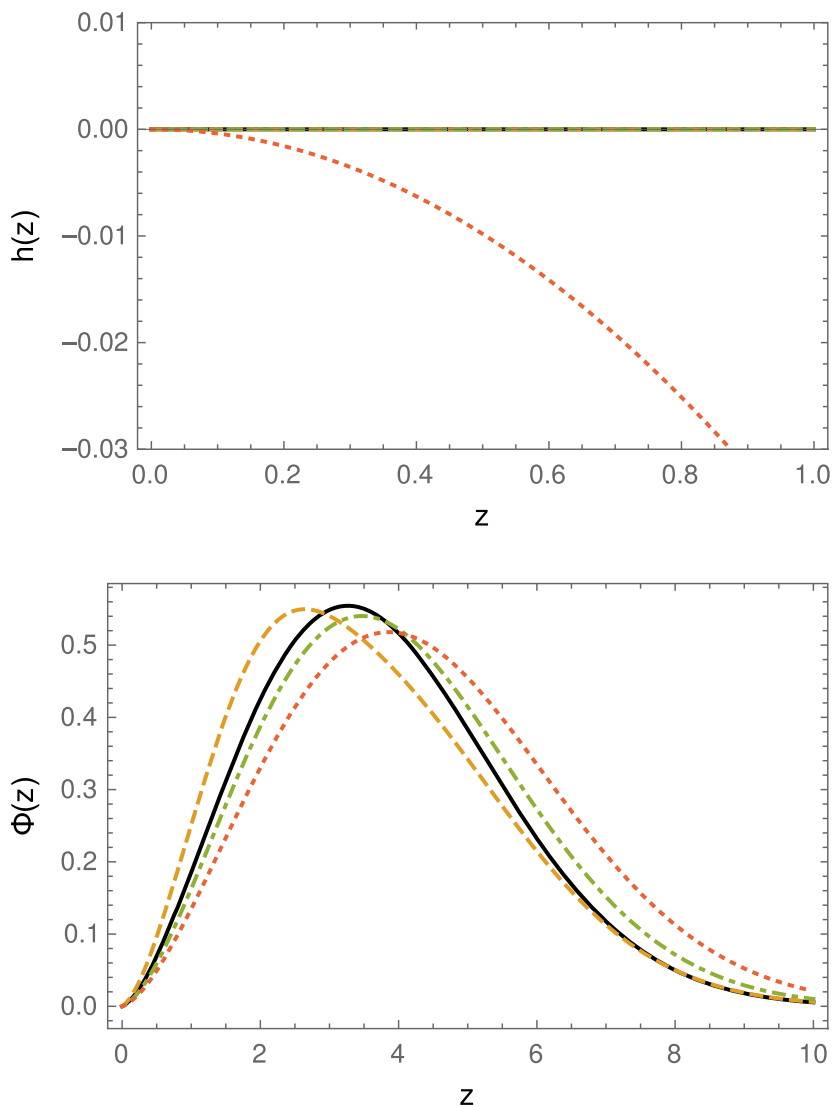

FIG. 1. The set of dilaton fields (left upper panel), the deformation function (right upper panel), holographic potentials (left lower panel), and AdS modes (right lower panel) considered in this work, where models 1, 2, 3, and 4 are denoted by black, dashed, dot-dashed, and dotted lines, respectively. 
field/operator matching condition via the conformal dimension associated to the bulk fields [23].

Thus, for a general asymptotically AdS space we have the expression for the two-body LF wave function

$$
\left|\tilde{\psi}_{q_{1} \bar{q}_{2}}(x, \zeta)\right|^{2}=A^{2} x(1-x) \frac{|\Phi(\zeta)|^{2}}{2 \pi \zeta},
$$

which in terms of $x$ and $b$ is written as

$\left|\tilde{\psi}_{q_{1} \bar{q}_{2}}(x, b)\right|^{2}=A^{2} \frac{\sqrt{x(1-x)}}{2 \pi b}|\Phi(\sqrt{x(1-x)} b)|^{2}$,

This means that the expression (11) with $g\left(Q^{2}, x\right)=1$ is general and valid to obtain a two-parton holographic LFWF for other models different from the usual soft-wall or hardwall model.
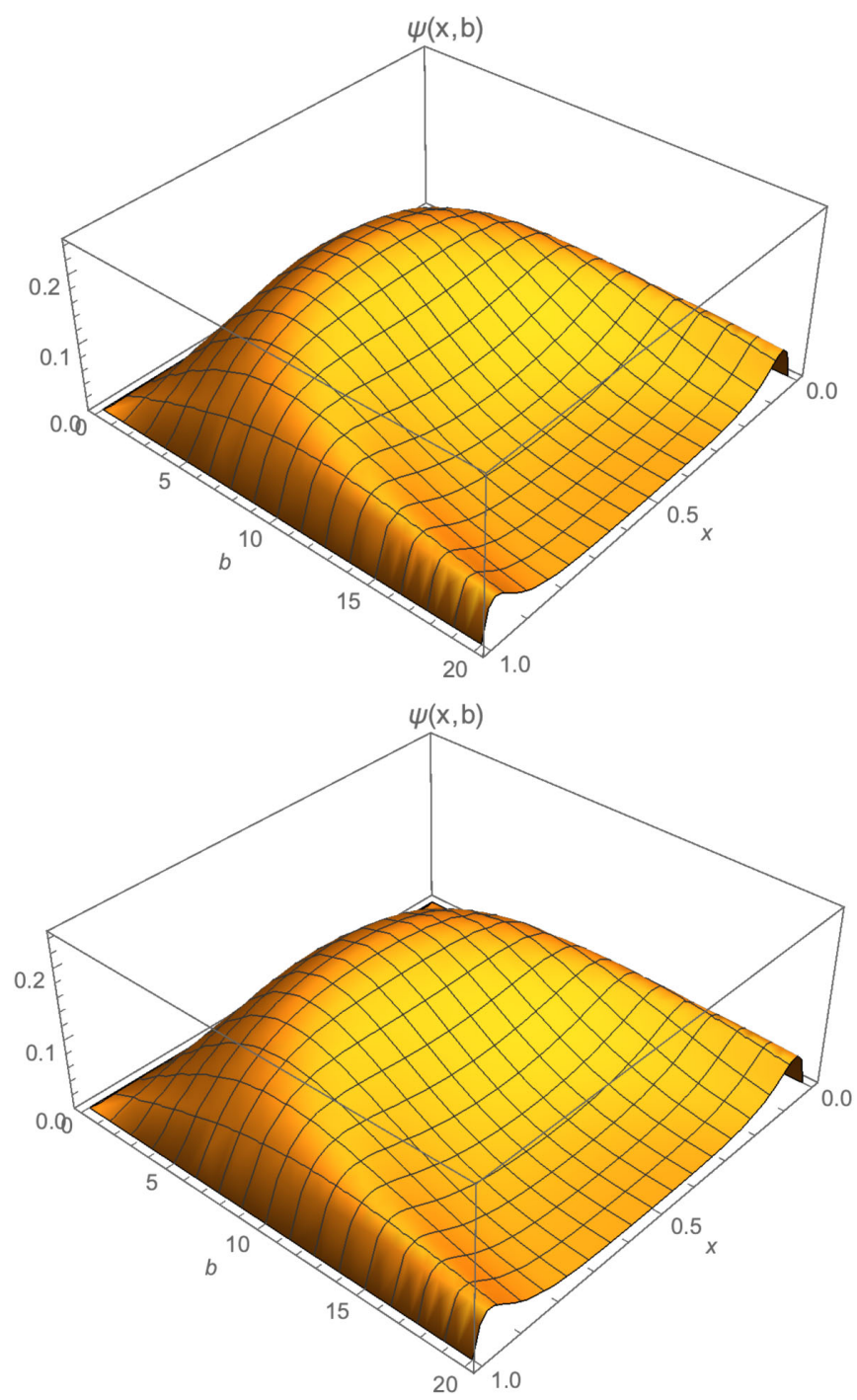

\section{EXAMPLES}

In this section we compare the LFWFs obtained for different dilatons. The recipe is as follows: once we have defined the dilaton and the AdS-like warp factor, we construct the holographic potential for scalar modes defined by

$$
\begin{aligned}
V(z)= & \frac{15}{4 z^{2}}+\frac{1}{4}\left[\phi^{\prime}(z)^{2}+9 h(z)^{2}\right]-\frac{3}{2} \phi^{\prime}(z) h^{\prime}(z) \\
& -\frac{1}{2}\left[\phi^{\prime \prime}(z)-3 h^{\prime \prime}(z)\right] \\
& -\frac{3}{2 z}\left[\phi^{\prime}(z)-3 h^{\prime}(z)\right]+\frac{e^{2 h(z)} M_{5}^{2} R^{2}}{z^{2}}
\end{aligned}
$$

where $M_{5}^{2} R^{2}$ is the bulk mass associated with the scalar modes. We fix $M_{5}^{2} R^{2}=-3$ in our analysis. This particular choice implies that we have dual scalar meson states.
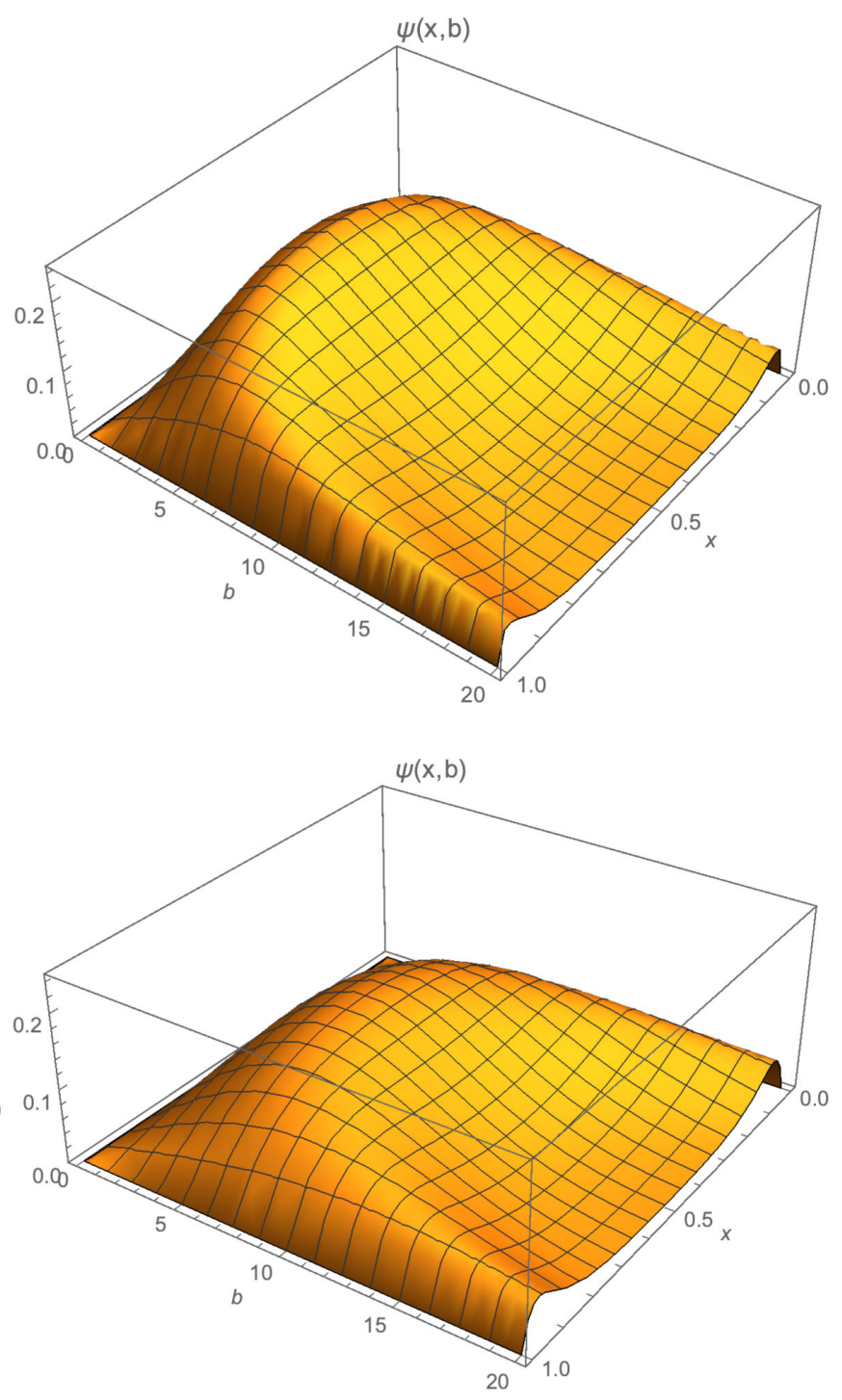

FIG. 2. The LFWF $\tilde{\psi}_{q_{1} \bar{q}_{2}}(x, b)$ for each model discussed: model 1 (left upper panel), model 2 (right upper panel), model 3 (left lower panel), and model 4 (right lower panel). 
The bulk Schrodinger-like modes are obtained by solving the Schrodinger-like EOM on the AdS side with the general holographic potential (25). With these modes, we can construct the two-body LF wave function (24).

We consider four different models from the AdS/QCD literature, characterized by the specific form of the dilaton field and the deformation function used. A summary of these models can be found in Table I.

Notice that in the light-cone applications, the parameters involved in the wave functions are fixed by considering constraints related to the phenomenology studied. But here, in order to compare the shapes of the LFWFs in different cases, we need to consider a parameter set that produces the same mass for the ground state calculated from the Schrodinger-like EOM on the AdS side. A summary of the parameters used in each model is shown in Table I.

In Fig. 1 we show a comparison between the four dilatons and deformation functions used in this paper, with their corresponding holographic potentials, given by the general expression (25), and the AdS Schrodinger-like modes calculated from such potentials.

In Fig. 2 we plot the LFWFs calculated for each of the four models discussed in this paper.

\section{CONCLUSIONS}

The relation between AdS modes and the LFWF is an interesting topic that has been restricted to the hard-wall [1] and soft-wall models with a quadratic dilaton [2] or phenomenological modifications on the QCD side $[3,15,16]$.

There exist plenty of AdS/QCD models that use different dilatons, or different asymptotically AdS geometries that try to catch many of the aspects of hadronic phenomenology that the standard hard-wall or soft-wall models with quadratic dilaton do not address. The LFWFs of these sorts of AdS/QCD models have not been studied because the matching procedure-which allows us to compare form factors on both sides and extract the LFWF in terms of AdS modes-was not discussed before in the specialized literature. Therefore, the approach considered here could be interesting because it allows to compute the LFWFs associated with these AdS/QCD approaches.

The key point to calculating the LFWFs related to AdS modes is to properly know the distribution $g\left(x, Q^{2}\right)$ defined in the expression (10). As it was discussed in Sec. III, for the large- $Q^{2}$ case this distribution is equal to one. Thus, we can obtain a two-body holographic LFWF for a wide variety of AdS/QCD models.

In the main part of this work, we focused our attention on the simplification of the equations of motion for the vector massless field, dual to photons on the AdS side. We noticed that for a wide range of AdS/QCD models we have $g\left(Q^{2}, x\right)=1$.

In the Appendix we discuss a different approach relating $g\left(x, Q^{2}\right)$ with the so-called Hankel transforms of the current
$J\left(z, Q^{2}\right)$. Then we use this idea in hard-wall and soft-wall models with quadratic dilaton models.

\section{ACKNOWLEDGMENTS}

We wish to acknowledge the financial support provided by FONDECYT (Chile) under Grants No. 1180753 (A. V.) and No. 3180592 (M. A. M. C.).

\section{APPENDIX A: EXTRACTION OF $g\left(Q^{2}, x\right)$ FROM $J\left(Q^{2}, z\right)$}

In order to use the expression (10) for different AdS/ QCD models, we need to compute the $g\left(Q^{2}, x\right)$ function used in the current expression (9). To do so, we consider the change of variable

$$
\alpha=\sqrt{\frac{1-x}{x}}
$$

in Eq. (9), which yields the following result:

$$
J\left(Q^{2}, z\right)=\int_{0}^{\infty} d \alpha \frac{2 \alpha}{\left(1+\alpha^{2}\right)^{2}} g(\alpha) J_{0}(\zeta Q \alpha) .
$$

Notice that the expression above looks like the Hankel transform of order zero. Therefore, by using the orthogonality relation

$$
\int_{0}^{\infty} J_{\nu}(k r) J_{\nu}\left(k^{\prime} r\right) r d r=\frac{1}{k} \delta\left(k-k^{\prime}\right)
$$

it is possible to find an expression for $g\left(Q^{2}, \alpha\right)$ as follows:

$\frac{2}{Q^{2}\left(1+\alpha^{2}\right)^{2}} g\left(Q^{2}, \alpha\right)=\int_{0}^{\infty} J\left(Q^{2}, z\right) J_{0}(z Q \alpha) z d z$.

The last expression allows us to get the distribution $g\left(Q^{2}, x\right)$ associated with a $J\left(Q^{2}, z\right)$ for different AdS/QCD models. Let us prove this expression in the context of the hard-wall and soft-wall models.

\section{Example 1: Hard-wall model}

In this case, the current has the following form:

$$
J(Q, z)=z Q K_{1}(z Q) .
$$

By using this current in the integral appearing in Eq. (A4) we can infer that $g\left(Q^{2}, \alpha\right)=g\left(Q^{2}, x\right)=1$, obtaining the well-known integral representation

$J(Q, z)=z Q K_{1}(z Q)=\int_{0}^{1} d x J_{0}\left(\zeta Q \sqrt{\frac{1-x}{x}}\right)$.

which is the representation used in Ref. [1] for the hardwall model. 


\section{Example 2: Traditional soft-wall model}

In this case, the current is written in terms of the Tricomi function as

$$
J\left(Q^{2}, z\right)=\Gamma\left(1+\frac{Q^{2}}{4 \kappa^{2}}\right) U\left(\frac{Q^{2}}{4 \kappa^{2}}, 0, \kappa^{2} z^{2}\right)
$$

In the large- $Q^{2}$ limit, where $Q^{2} \gg 4 \kappa^{2}$, it is possible to deduce that

$$
J\left(Q^{2}, z\right) \rightarrow z Q K_{1}(z Q) .
$$

Therefore, the integral representation for $J\left(Q^{2}, z\right)$ is the same as the one in the hard-wall case. But if we use the expression (A7) in Eq. (A4), we obtain

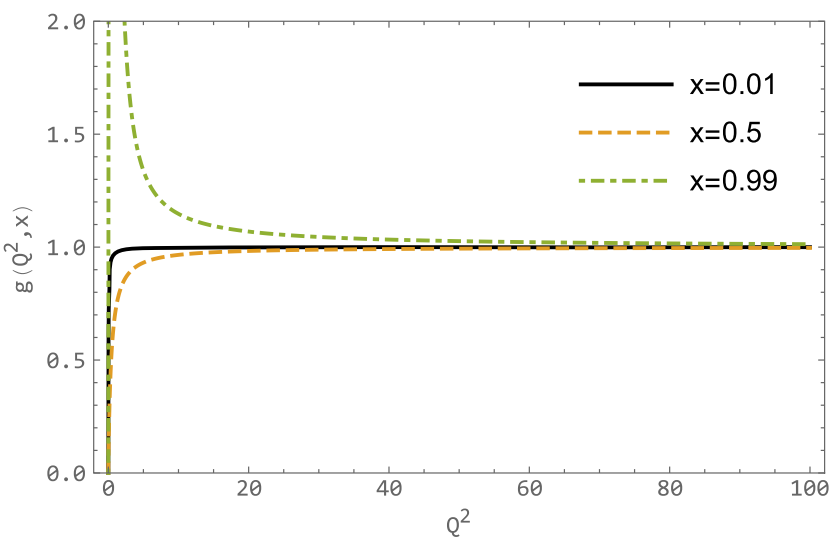

FIG. 3. The behavior of $g\left(Q^{2}, x\right)$ as a function of $Q^{2}$ for different $x$ values. In all of the cases plotted, we observe $g\left(Q^{2} \rightarrow \infty, x\right)=1$.

$$
g\left(Q^{2}, \alpha\right)=\frac{\left(\alpha^{2}+1\right)^{2} Q^{2}}{4 \kappa^{2}}\left(\left(\alpha^{2}+1\right) 2^{-\frac{Q^{2}}{2 \kappa^{2}}} Q^{\frac{Q^{2}}{2 \kappa^{2}}} \kappa^{-\frac{Q^{2}}{2 \kappa^{2}}} e^{\frac{\alpha^{2} Q^{2}}{4 \kappa^{2}}} \alpha^{2\left(\frac{Q^{2}}{4 \kappa^{2}}-1\right)} \Gamma\left(1-\frac{Q^{2}}{4 \kappa^{2}}, \frac{Q^{2} \alpha^{2}}{4 \kappa^{2}}\right)-1\right) .
$$

However, by using Eq. (A1) we have

$$
g\left(Q^{2}, x\right)=\frac{Q^{2}}{4 \kappa^{2}}\left(\frac{1}{x}\right)^{2}\left(\frac{2^{-\frac{Q^{2}}{2 \kappa^{2}}} Q^{\frac{Q^{2}}{2 \kappa^{2}} \kappa^{-}} \frac{Q^{2}}{2 \kappa^{2}} e^{\frac{Q^{2}(1-x)}{4 \kappa^{2} x}}\left(\frac{1-x}{x}\right)^{\frac{Q^{2}}{4 \kappa^{2}}-1} \Gamma\left(1-\frac{Q^{2}}{4 \kappa^{2}}, \frac{Q^{2}(1-x)}{4 x \kappa^{2}}\right)}{x}-1\right) .
$$

As we can infer from Fig. 3, the distribution $g\left(Q^{2}, x\right)$ goes to one at large $Q^{2}$, which is the limit where the valence contribution is dominant in the Fock expansion. Therefore, the result depicted in this work is the same as that presented in Ref. [2] and used by several authors.

[1] S. J. Brodsky and G. F. de Teramond, Phys. Rev. Lett. 96, 201601 (2006).

[2] S. J. Brodsky and G. F. de Teramond, Phys. Rev. D 77, 056007 (2008).

[3] S. J. Brodsky and G. F. de Téramond, Subnuclear series 45, 139 (2009).

[4] A. Vega, I. Schmidt, T. Branz, T. Gutsche, and V.E. Lyubovitskij, Phys. Rev. D 80, 055014 (2009).

[5] M. Ahmady, R. Sandapen, and N. Sharma, Phys. Rev. D 94, 074018 (2016).

[6] A. Bacchetta, S. Cotogno, and B. Pasquini, Phys. Lett. B 771, 546 (2017).

[7] Q. Chang, S. Xu, and L. Chen, Nucl. Phys. B921, 454 (2017).

[8] T. Liu and B.-Q. Ma, Phys. Rev. D 92, 096003 (2015).

[9] R. Swarnkar and D. Chakrabarti, Phys. Rev. D 92, 074023 (2015).

[10] A. Vega, I. Schmidt, T. Gutsche, and V. E. Lyubovitskij, Phys. Rev. D 93, 056001 (2016).

[11] S. Momeni and R. Khosravi, Eur. Phys. J. C 78, 805 (2018).
[12] M. Ahmady, C. Mondal, and R. Sandapen, Phys. Rev. D 100, 054005 (2019).

[13] N. Kaur and H. Dahiya, Eur. Phys. J. A 56, 172 (2020).

[14] M. Ahmady, S. Keller, M. Thibodeau, and R. Sandapen, Phys. Rev. D 100, 113005 (2019).

[15] S. Chabysheva and J. R. Hiller, Ann. Phys. (Amsterdam) 337, 143 (2013).

[16] T. Gutsche, V. E. Lyubovitskij, I. Schmidt, and A. Vega, Phys. Rev. D 89, 054033 (2014); 92, 019902(E) (2015).

[17] S. Hong, S. Yoon, and M. J. Strassler, J. High Energy Phys. 04 (2006) 003.

[18] T. Gutsche, V. E. Lyubovitskij, I. Schmidt, and A. Vega, Phys. Rev. D 86, 036007 (2012).

[19] T. Gutsche, V. E. Lyubovitskij, I. Schmidt, and A. Vega, Phys. Rev. D 87, 016017 (2013).

[20] T. Gutsche, V. E. Lyubovitskij, and I. Schmidt, Phys. Rev. D 101, 034026 (2020).

[21] T. Gutsche, V. E. Lyubovitskij, I. Schmidt, and A. Vega, Phys. Rev. D 91, 114001 (2015).

[22] T. Gutsche, V. E. Lyubovitskij, I. Schmidt, and A. Vega, Phys. Rev. D 85, 076003 (2012). 
[23] E. Witten, Adv. Theor. Math. Phys. 2, 253 (1998).

[24] A. Karch, E. Katz, D. T. Son, and M. A. Stephanov, Phys. Rev. D 74, 015005 (2006).

[25] D. Li and M. Huang, J. High Energy Phys. 11 (2013) 088.
[26] N. R. Braga and L. F. Ferreira, Phys. Lett. B 795, 462 (2019).

[27] E. Folco Capossoli, M. A. M. Contreras, D. Li, A. Vega, and H. Boschi-Filho, Chin. Phys. C 44, 064104 (2020). 